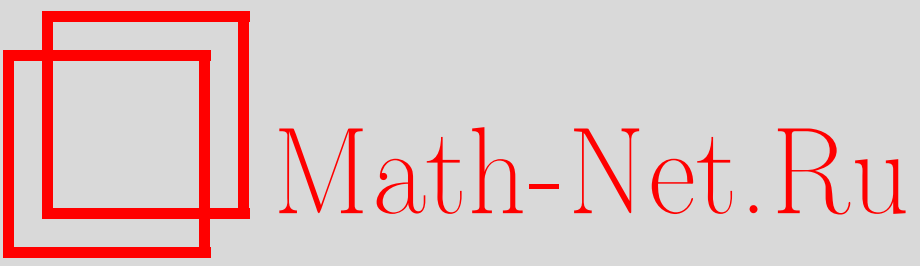

О. Н. Попов, Об одной конструкции модулей над кольцом многочленов в случае произвольного поля, УМН, 2004, том 59, выпуск 3, 175-176

DOI: https://doi.org/10.4213/rm751

Использование Общероссийского математического портала Math-Net.Ru подразумевает, что вы прочитали и согласны с пользовательским соглашением

http://www.mathnet.ru/rus/agreement

Параметры загрузки:

IP: 54.198 .67 .100

26 апреля 2023 г., 14:43:11 


\title{
ОБ ОДНОЙ КОНСТРУКЦИИ МОДУЛЕЙ НАД КОЛЬЦОМ МНОГОЧЛЕНОВ В СЛУЧАЕ ПРОИЗВОЛЬНОГО ПОЛЯ
}

\author{
О. Н. Попов
}

В работе [1] мш рассматривали следующую конструкцию модулей над кольцом многочленов. Пусть $A$ - конечномерная ассоциативная алгебра с 1 над полем $\mathbb{k}$ с базисом $e_{1}, \ldots, e_{d}$, а $\rho: A \rightarrow$ $M_{n}(\mathbb{k})$ - ее матричное представление, отвечающее $A$-модулю $M, \operatorname{dim}_{\mathbb{k}} M=n$. Зададим натуральное $l>1$ и рассмотрим кольцо многочленов $R=\mathbb{k}\left[x_{11}, \ldots, x_{d l}\right]$ и модуль $F_{l}(M)$ над ним, являющийся фактормодулем свободного $R$-модуля $R^{n}$ по подмодулю, порожденному столбцами матриц $\operatorname{Id}_{j}=\sum_{i} \rho\left(e_{i}\right) x_{i j}, j=1, \ldots, l$.

В работе исследовался вопрос, для каких алгебр получающиеся модули над кольцом многочленов - коэн-маколеевы или $F_{l}$ - точный функтор. Ответ был получен в случае совершенного поля $\mathbb{k}$, а в [2] были описаны алгебры над совершенньп полем, для которых неразложимые представления переходят в коэн-маколеевы модули. В данной работе мы даем ответ для произвольного поля, а также описываем, когда для данного представления получается коэн-маколеев модуль над кольцом многочленов.

Сначала дадим определение, восходящее к работам Адзумая [3], [4], в которых доказана равносильность большинства пунктов:

ПРЕДЛОЖЕНИЕ-ОПРЕДЕЛЕНИЕ. Конечномерная ассочиативная алгебра $A$ над $\mathbb{k}$ c eдиницей называется максимально центральной алгеброй, если выполнено одно из следующих эквивалентных условий:

1) $A$ - прямая сумма алгебр $A_{i}$, факторь которьх по радикалу просты $u \operatorname{dim}_{\mathbb{k}} A_{i} \leqslant$ $t_{i}^{2} \operatorname{dim}_{\mathbb{k}} Z\left(A_{i}\right)$, где $t_{i}^{2}-$ это ранг $A_{i} / \operatorname{rad} A_{i}$ над своим чентром и на самом деле имеет место равенство;

2) при проекции $A$ на любую ее факторалгебру $B$ чентр $A$ сюрвективно отображается на чентр $B$;

3) алгебра $\bar{A}$, полученная из $A$ переходом к алгебраическому замыканию основного поля, - прямая сумма матричных алгебр над своими чентрами;

4) алгебра $A \otimes_{\mathbb{k}} L$, где $L$ - произвольное расширение поля $\mathbb{k}$, удовлетворяет условию 1);

5) $A$ - прямая сумма таких алгебр $A_{i}$ с чентрами $Z_{i}$, что $A_{i}$ - свободнье $Z_{i}$-модули и $\operatorname{End}_{Z_{i}} A_{i}=A_{i} \otimes Z_{i} A_{i}^{0}$

6) $A$ - алгебра Адзумая над своим иентром $Z$, т.е. [5; гл. VI, §1, определение 1.5] такой проективный $Z$-модуль, что для любого простого идеала $\mathfrak{p} \subset Z A \otimes_{Z} k(\mathfrak{p})$ - чентральная простая алгебра над $k(\mathfrak{p})$.

Если основное поле совершенно, то әти условия әквивалентны следующему:

7) $A=\bigoplus_{i} S_{i} \otimes_{Z\left(S_{i}\right)} K_{i}$, где $S_{i}$ - простые алгебры над $\mathbb{k}$, а $K_{i}$ - конечномерные коммутативные алгебры над соответствующими $Z\left(S_{i}\right)$ ( $A$ - "линейная комбинация простых алгебр с коммутативными коэффициентами”).

Будем назьвать максимально центральную алгебру равноразмерной, если алгебра $\bar{A}$ из условия 3) - прямая сумма матричных алгебр одного и того же ранга над своими центрами или, что то же самое, $\bar{A}$ - матричная алгебра над конечномерной коммутативной.

Тогда основной резулттат формулируется так:

Теорема. Если $A$ - максимально чентральная алгебра, то для любого $l>1$ все неразложимые $A$-модули переходят в коэн-маколеевы $R$-модули и $F_{l}$ - точный вполне строгий функтор в категорию градуированных $R$-модулей, а если при этом $A$ равноразмерна, то и все А-модули переходят в коэн-маколеевы. Наоборот, если для некоторого

Работа выполнена при частичной поддержке Российского фонда фундаментальных исследований (грант № 02-01-00468).

(C) О.н. Попов 2004 
$l>1$ либо функтор $F_{l}$ точен, либо для всех неразложимых модулей $M$ модули $F_{l}(M)$ коэн-маколеевы, то $A$ - максимально чентральная алгебра, а если отбросить условие неразложимости, то $A$ - равноразмерная максимально чентральная алгебра. Более того, для произвольной (ассоциативной с 1 ) алгебрь $A$ для некоторого $A$-модуля $M$ и некоторого $l>1$ модуль $F_{l}(M)$ коэн-маколеев, если и только если $A / \operatorname{ann} M$ - равноразмерная максимально центральная алгебра.

Автор хотел бы поблагодарить А. А. Герко, давшего ему мотивацию доделать эту работу.

\section{СПИСОК ЛИТЕРАТУРЫ}

[1] О. Н. Попов // Матем. сб. 2002. Т. 193. № 3. С. 115-134. [2] О. Н. Попов // УМН. 2003. T. 58. № 2. C. 173-174. [3] G. Azumaya, T. Nakayama// Japan J. Math. 1948. V. 19. № 4. P. 263-273. [4] G. Azumaya // Nagoya Math. J. 1951. V. 2. Р. 119-150. [5] Ю. И. Манин. Кубические формы. М.: Наука, 1972.

Московский государственньй

Принято редколлегией университет им. М.В. Ломоносова 01.04.2004 